\title{
Análise da qualidade da água do rio dos Índios, Presidente Getúlio, SC, Brasil
}

A água é um recurso essencial à todas as atividades humanas, fato que torna relevante o estudo da disponibilidade hídrica para tais atividades, bem como a sua qualidade. O objetivo do presente estudo consistiu em avaliar a qualidade da água do rio dos Índios, localizado no município de Presidente Getúlio (SC, Brasil). As amostras de água foram avaliadas por meio de ensaios físico-químicos (oxigênio dissolvido-OD, temperatura, nitrato, nitrito, turbidez, $\mathrm{pH}$, cor verdadeira e $\mathrm{DBO}$ ) e série de metais (alumínio, manganês, antimônio, arsênio, cádmio, chumbo, cobalto, cromo, estrôncio, ferro, molibdênio, prata, tálio, vanádio e zinco). 0 estudo compreendeu cinco pontos de amostragem ao longo do corpo hídrico, abrangendo a zona rural, perímetro urbano e zona limítrofe entre a zona rural e perímetro urbano. A Análise de Variância (ANOVA) possibilitou a verificação de quais parâmetros mais variaram em relação aos pontos de amostragem durante o período das coletas. Os resultados dos parâmetros físico-químicos (DBO5 > $5 \mathrm{mg} \mathrm{L-1)} \mathrm{e} \mathrm{análise} \mathrm{de} \mathrm{metais} \mathrm{(alumínio,} \mathrm{manganês,} \mathrm{arsênio} \mathrm{e} \mathrm{ferro)} \mathrm{não} \mathrm{atendem} \mathrm{aos} \mathrm{limites}$ estabelecidos pela Resolução CONAMA 357/2005. Os resultados apresentados neste estudo evidenciam possíveis fontes de contaminação do rio dos índios resultantes das atividades humanas de urbanização, industrial e agrícola nas proximidades da região.

Palavras-chave: Qualidade da água; Rio dos Índios; Análises físico-químicas; Metais.

\section{Water quality analysis of Índios river, Presidente Getúlio, SC, Brazil}

\begin{abstract}
Water is an essential resource to all human activities. This fact makes it relevant to study its availability as well as its quality. The objective of this work is to evaluate the water quality of Rio dos Índios river. This watercourse is located in the municipality of Presidente Getúlio, SC-Brazil. The water samples were analyzed for physico-chemical tests (dissolved oxygen-DO, temperature, nitrate, nitrite, turbidity, $\mathrm{pH}$, true color and BOD5) and series of metals (aluminum, manganese, antimony, arsenic, cadmium, lead, cobalt, chromium, strontium, iron, molybdenum, silver, thallium, vanadium and zinc). The survey was carried out in five sampling points through the watercourse comprehending the rural zone, the urban perimeter, and the borderline between both. Methods statistical data (ANOVA), on the other hand, enabled the verification of which parameters most varied in relation to the sampling points during the collection period. The results for physicochemical parameter (BOD5 > $5 \mathrm{mg} \mathrm{L}-1$ ) and metals (aluminum, manganese, arsenic and iron) surpassed the thresholds stablished by the CONAMA resolution $357 / 2005$. The results presented within this work point out potential sources of contamination to the Rio dos índios river as a resultant from the urbanization, industrial and agricultural activities near the region.
\end{abstract}

Keywords: Water quality; Índios river; Physico-chemical analysis; Metals.

Topic: Engenharia Ambiental

Reviewed anonymously in the process of blind peer.
Received: 03/01/2021

Approved: 28/01/2021
Natânie Bigolin Narciso (iD

Universidade do Estado de Santa Catarina, Brasil

http://lattes.cnpq.br/1014264106986785

http://orcid.org/0000-0001-8099-4989

natanie_narciso@outlook.com

\section{Karina Lunelli (iD)}

Universidade do Estado de Santa Catarina, Brasil

http://lattes.cnpq.br/3691167988338673

http://orcid.org/0000-0002-6595-3318

lunellikari@gmail.com

Eduardo Elias Engesser (10)

Universidade do Estado de Santa Catarina, Brasil

http://lattes.cnpq.br/0251039989165945

http://orcid.org/0000-0002-6449-9109

eduardo.engesser@edu.udesc.br

\author{
Luciano André Deitos Koslowski (iD) \\ Universidade do Estado de Santa Catarina, Brasil \\ http://lattes.cnpq.br/4753037489573483 \\ http://orcid.org/0000-0003-2917-8681 \\ luciano.koslowski@udesc.br \\ Jonathan Tenório de Lima (iD) \\ Universidade do Estado de Santa Catarina, Brasil \\ http://lattes.cnpq.br/3998560860341516 \\ http://orcid.org/0000-0002-0556-8530 \\ jonathantenorio88@gmail.com
}

Referencing this:

NARCISO, N. B.; LUNELLI, K.; ENGESSER, E. E.; KOSLOWSKI, L. A. D.; LIMA, J. T.. Análise da qualidade da água do rio dos Índios, Presidente Getúlio, SC, Brasil. Revista Ibero Americana de Ciências Ambientais, v.12, n.1, p.362-374, 2021. DOI: http://doi.org/10.6008/CBPC2179$\underline{6858.2021 .001 .0030}$ 


\section{INTRODUÇÃO}

A água constitui um elemento essencial para a existência dos seres vivos sendo, portanto, um recurso natural insubstituível. Neste aspecto, a água apresenta considerável importância nas atividades humanas notadamente o abastecimento público e industrial, irrigação agrícola, produção de energia elétrica e as atividades de lazer e recreação (ALVES et al., 2008; CALEGARI et al., 2015).

Os ecossistemas aquáticos sofrem impactos consideráveis pelas atividades humanas e, de forma direta ou indireta, produzem alterações em estuários e águas costeiras, redução de vegetação natural e alteração das características naturais de drenagem (LI et al., 2017; OLIVEIRA et al., 2018; LACH et al., 2020). Portanto, a exploração dos ecossistemas aquáticos de forma não sustentável pela sociedade humana tem contribuído para a redução da qualidade da água e sua escassez (PEREIRA et al., 2020).

A presença de contaminantes nos corpos hídricos provenientes da introdução de poluentes da indústria e de esgotos domésticos tem motivado uma redução de organismos aquáticos e alteração do sistema ecológico nestes locais (GOIS et al., 2016; YAN et al., 2018).

Conforme Marcuzzo et al. (2011), a bacia do rio dos Índios apresenta área formada pelo rio correspondente a $628,5 \mathrm{~km}^{2}$, sendo, portanto, uma sub-bacia da bacia hidrográfica do rio Itajaí Açu constituída por grandes regiões de baixa altitude suscetível a extensos alagamentos. $O$ rio dos Índios (extensão de 48,10 km lineares, gradiente de $276 \mathrm{~m}$ distribuído, e declive de 0,17 m.km-1) ocupa toda a extensão do município de Presidente Getúlio, região situada na microrregião do Alto Vale do Itajaí, no estado de Santa Catarina.

O rio dos Índios abrange o perímetro rural e urbano do município de Presidente Getúlio - SC. De acordo com os dados da AMAVI (2019), a principal contribuição econômica do município de Presidente Getúlio se refere ao setor agrícola $(23,9 \%)$ e pela indústria alimentícia $(17,1 \%)$.

Nesta perspectiva, o monitoramento regular da qualidade da água constitui em um dispositivo necessário para o acompanhamento de dados quantitativos em relação à gestão da água (SCOTT et al., 2017; LACH et al., 2020) e permite avaliar as influências exercidas pelas atividades humanas e por fatores naturais sobre o ambiente, saúde e bem-estar da população (BARRETT et al., 2016)

Nas áreas onde a economia é fortemente baseada em atividades da agroindústria, a água representa um consumo de $80 \%$ da demanda necessária para atender o setor produtivo, tornando a exploração deste recurso hídrico insustentável (VERGINE et al., 2017). Da mesma forma, o efluente gerado apresenta considerável concentração de matéria orgânica, sólidos em suspensão, óleos e graxas e nutrientes (BUSTILLO-LECOMPTE et al., 2015; CREMONINI et al., 2020). Cremonini et al. (2020) reportam a relação entre o elevado teor de gordura, corantes e aditivos empregados nos processos agroindustriais e sua não biodegradabilidade associada a uma estrutura orgânica complexa e de difícil degradação empregando técnicas de tratamento de efluentes convencionais.

Neste ínterim, diversas pesquisas têm sido desenvolvidas na quantificação de metais pesados em bacias hidrográficas (MACHADO et al., 2017; SILVA et al., 2018; PEREIRA et al., 2019), considerando o impacto 
ambiental relacionada a possíveis atividades econômicas e identificação de possíveis fontes poluidoras SANTOS, 2015; EIDT et al., 2016; OLIVEIRA et al., 2017).

Nesse sentido, esse trabalho teve como propósito avaliar a qualidade da água do rio dos Índios utilizando análises laboratoriais físico-químicas bem como a presença de metais. Portanto, o estudo será estendido tanto para a zona rural como para o perímetro urbano do munícipio de Presidente Getúlio/SC, sendo possível mensurar as atividades antrópicas decorrente da ocupação urbana, industrial e agrícola.

\section{METODOLOGIA}

A qualidade da água do rio dos Índios foi avaliada por meio de determinações de parâmetros físicos, químicos e microbiológicos. A área de estudo está situada na bacia hidrográfica do rio dos Índios na região do Alto Vale do Itajaí, no município de Presidente Getúlio, Santa Catarina. O município de Presidente Getúlio localiza-se na latitude S $27^{\circ} 2^{\prime} 48^{\prime \prime}$ e longitude $\mathrm{O} 49^{\circ} 37^{\prime} 21^{\prime \prime}$, com população estimada de 17.471 mil habitantes, altitude de $242 \mathrm{~m}$, área total de 297,60 km² e faz limite com as cidades de Dona Emma, Ibirama, José Boiteux, Laurentino, Lontras, Rio do Oeste e Rio do Sul. A região hidrográfica apresenta pluviosidade anual de cerca de $1550 \mathrm{~mm}$, altitude de $242 \mathrm{~m}$, relevo predominantemente montanhoso em clima subtropical úmido com invernos frios e temperatura média anual de $18^{\circ} \mathrm{C}$ (SANTA CATARINA, 2020).

A Figura 1 abaixo ilustra a localização dos pontos amostrais (P) e a malha municipal, empregando o sistema de coordenadas geográficas por meio do software QGIS Desktop 2.18.18, conforme dados disponibilizados pela EPAGRI/CIRAM (2020).

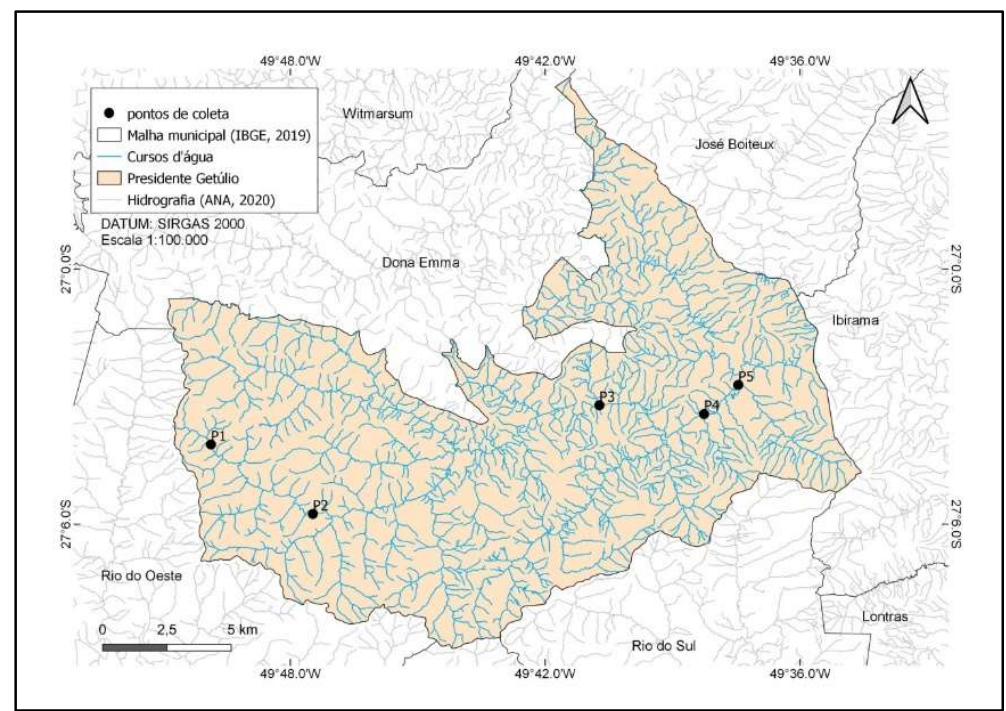

Figura 1: Localização dos pontos de coleta das amostras de água, hidrografia, malha municipal e curso d'água do rio dos Índios, Presidente Getúlio, SC.

Os cinco pontos amostrais de análise da água foram avaliados criteriosamente, abrangendo a zona rural do município (P1 e P2), zona limítrofe entre a área rural e urbana (P3) e perímetro urbano (P4 e P5) conforme sumarizado na Tabela 1.

As amostras de água foram coletadas por amostragem simples em triplicata para cada um dos pontos, em intervalos de 40 dias compreendido entre os meses de agosto a dezembro de 2019. As amostras 
de água foram coletadas empregando frascos de borosilicato âmbar estéreis de $500 \mathrm{~mL}$ (Laborquim), empregando a técnica de refrigeração sem congelamento $\left(4^{\circ} \mathrm{C}\right)$ minimizando uma potencial volatilização de gases dissolvidos e reduzindo a atividade microbiológica. As análises foram realizadas em conformidade com a NBR 9898/87 (ABNT, 1987). A Figura 2 ilustra os pontos de amostragem quanto à sua localização na área rural e urbana.

Tabela 1: Localização geográfica e atividade econômica relacionada a cada ponto de amostragem (P) do rio dos Índios no município de Presidente Getúlio-SC.

\begin{tabular}{|l|l|l|}
\hline Pontos & Coordenadas geográficas & Atividade econômica predominante \\
\hline P1 & $27^{\circ} 04^{\prime} 07.65^{\prime \prime} \mathrm{S}$ & Agrossilvipastoril \\
& $49^{\circ} 49^{\prime} 52.12^{\prime \prime} \mathrm{W}$ & Agrossilvipastoril \\
\hline P2 & $27^{\circ} 05^{\prime} 45.69^{\prime \prime} \mathrm{S}$ & \\
& $49^{\circ} 47^{\prime} 28.16^{\prime \prime} \mathrm{W}$ & Agropecuária, industrial e atividades urbanas \\
\hline P3 & $27^{\circ} 03^{\prime} 12.28^{\prime \prime} \mathrm{S}$ & \\
& $49^{\circ} 40^{\prime} 43.33^{\prime \prime} \mathrm{W}$ & Agropecuária, industrial e atividades urbanas \\
\hline P4 & $27^{\circ} 03^{\prime} 24.71^{\prime \prime} \mathrm{S}$ & \\
& $49^{\circ} 38^{\prime} 15.54^{\prime \prime} \mathrm{W}$ & Industrial, serviços e atividades urbanas \\
\hline
\end{tabular}

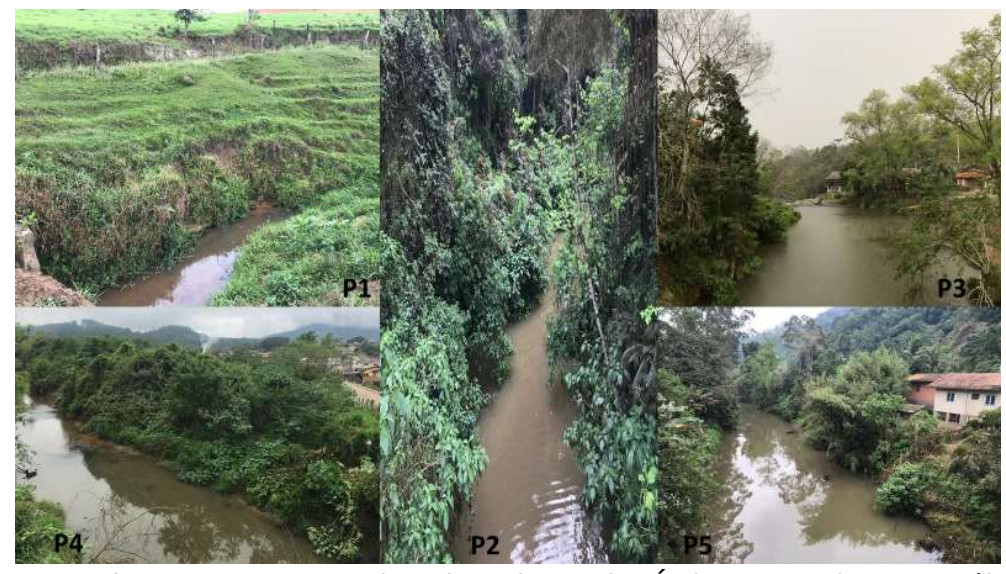

Figura 2: Imagens correspondentes aos pontos de coleta do rio dos Índios, Presidente Getúlio (SC) na área rural do município (P1 e P2), área limítrofe da área rural/urbana (P3) e área urbana do município (P4 e P5).

Tabela 2: Métodos utilizados na realização dos ensaios laboratoriais.

\begin{tabular}{|c|c|}
\hline Parâmetro & Referência e Método \\
\hline Oxigênio Dissolvido & SMEEWW $^{(1)} 4500-0$ G. \\
\hline Temperatura & SMEEWW 2550 \\
\hline Nitrogênio Total & DS/EN ISO ${ }^{(2)} 11905-1$ \\
\hline Nitrato & $\mathrm{APHA}^{(3)} 4500-\mathrm{NO}_{3}$ \\
\hline Nitrito & APHA 4500- $\mathrm{NO}_{2}$ \\
\hline Turbidez & SMEEWW 2130B \\
\hline Alumínio & SMEWW - 3030E e 3120B \\
\hline Manganês & SMEWW - 3030E e 3120B \\
\hline Antimônio & SMEWW - 3030E e 3120B \\
\hline Arsênio & SMEWW - 3030E e 3120B \\
\hline Cádmio & SMEWW - 3030E e 3120B \\
\hline Chumbo & SMEWW - 3030E e 3120B \\
\hline Cobalto & SMEWW - 3030E e 3120B \\
\hline Cromo & SMEWW - 3030E e 3120B \\
\hline Estrôncio & SMEWW - 3030E e 3120B \\
\hline Ferro & SMEWW - 3030E e 3120B \\
\hline Molibdênio & SMEWW - 3030E e $3120 B$ \\
\hline Prata & SMEWW - 3030E e 3120B \\
\hline Tálio & SMEWW - 3030E e 3120B \\
\hline Vanádio & SMEWW - 3030E e 3120B \\
\hline Zinco & SMEWW - 3030E e 3120B \\
\hline $\mathrm{pH}$ & SMEWW- $4500 \mathrm{H}^{+} . \mathrm{B}$ \\
\hline Cor verdadeira & SMEWW-2120D \\
\hline $\mathrm{DBO}_{5}$ & SMEWW-5210D \\
\hline
\end{tabular}

(1) Standard Methods for the Examination of Water and Wastewater, 2012. (2) International Organization for Standardization (DS/EN ISO, 1997).

(3) American Public Health Association, 2012. 
Os parâmetros físico-químicos foram avaliados seguindo os procedimentos descritos no Standard Methods for the Examination of Water and Wastewater da American Public Health Association (APHA, 2012) e International Organization for Standardization (ISO 11905-1, 1997) conforme sumarizado na Tabela 2. Os seguintes ensaios físico-químicos e microbiológicos foram realizados: determinação de Oxigênio Dissolvido - OD pelo método eletrométrico (Oxímetro Logen Scientific, modelo L50D-4000 Série 7420), pH via método eletrométrico (Hanna HI 3221), cor verdadeira (Hach DR/2010), turbidez (Hanna HI 93703), quantificação de nitrato, nitrito e nitrogênio total pelo método colorimétrico (Merck Spectroquant Multy), demanda bioquímica de oxigênio- $\mathrm{DBO}_{5}$ (Hach, Modelo HQ30D) e quantificação de metais por meio de análise via Espectrômetro de Emissão Óptica com Plasma Acoplado Indutivamente (ICP-OES Optima 8300 Perkin-Elmer).

\section{Análise dos dados}

Os dados obtidos em relação às análises físico-químicas foram avaliados empregando modelos estatísticos utilizando análise de variância (ANOVA) e comparação das médias pelo teste de Tukey $(p \leq 0.05)$ empregando o programa Statistica para Windows, versão 7.0, Statsoft.

\section{RESULTADOS E DISCUSSÃO}

Os valores das análises físico-químicos da água do rio dos Índios foram obtidos em cinco pontos de amostragem (P) e, confrontados com os valores estabelecidos pelo Conselho Nacional do Meio Ambiente CONAMA no 357 de 2005 (CONAMA, 2005). A respeito das classes de qualidade do curso d'água, o rio dos Índios enquadra-se como classe II segundo Portaria no 024/79, que rege o enquadramento dos cursos d'água do estado de Santa Catarina (FATMA, 1979).

\section{Oxigênio Dissolvido e Temperatura}

A Figura 3 apresenta os valores obtidos para oxigênio dissolvido (OD) e temperatura em todos os cinco pontos de amostragem analisados na bacia hidrográfica do rio dos Índios.

Os dados obtidos da tabela 3 foram estatisticamente avaliados por análise de variância (ANOVA) e a comparação de médias pelo teste de Tukey a $5 \%$, sendo que os parâmetros analisados (oxigênio dissolvido e temperatura) não diferem entre si estatisticamente $(p<0,05)$.

A análise da água do rio dos Índios em relação aos seus cinco pontos de amostragem indica valores médios e desvio padrão (DP) compreendidos entre $9,04 \mathrm{mg} \mathrm{L}^{-1} \pm 0,56$ (P1) a 7,77 $\mathrm{mg} \mathrm{L}^{-1} \pm 2,04$ (P5) para o oxigênio dissolvido. Portanto, conforme estabelecido pela Resolução do Conama n 357/05 as análises da água do rio dos Índios demonstraram que, para OD, apresentam valores próximos e acima do valor mínimo permitido para classe $2\left(>5 \mathrm{mg} \mathrm{L}^{-1}\right)$.

O oxigênio constitui um dos parâmetros mais utilizados para a avaliação dos ecossistemas aquáticos, fornecendo um possível efeito de contaminação de efluentes domésticos em contato com a biota do rio com redução da disponibilidade de oxigênio (AMORIM et al., 2017). Lunelli et al. (2019) realizaram monitoramento da qualidade da água do rio dos Índios entre os meses de abril e maio de 2018 com valores 
observados de OD compreendidos entre 5,00 e 6,00 $\mathrm{mg} \mathrm{L}^{-1}$, corroborando com os dados obtidos nesta pesquisa.

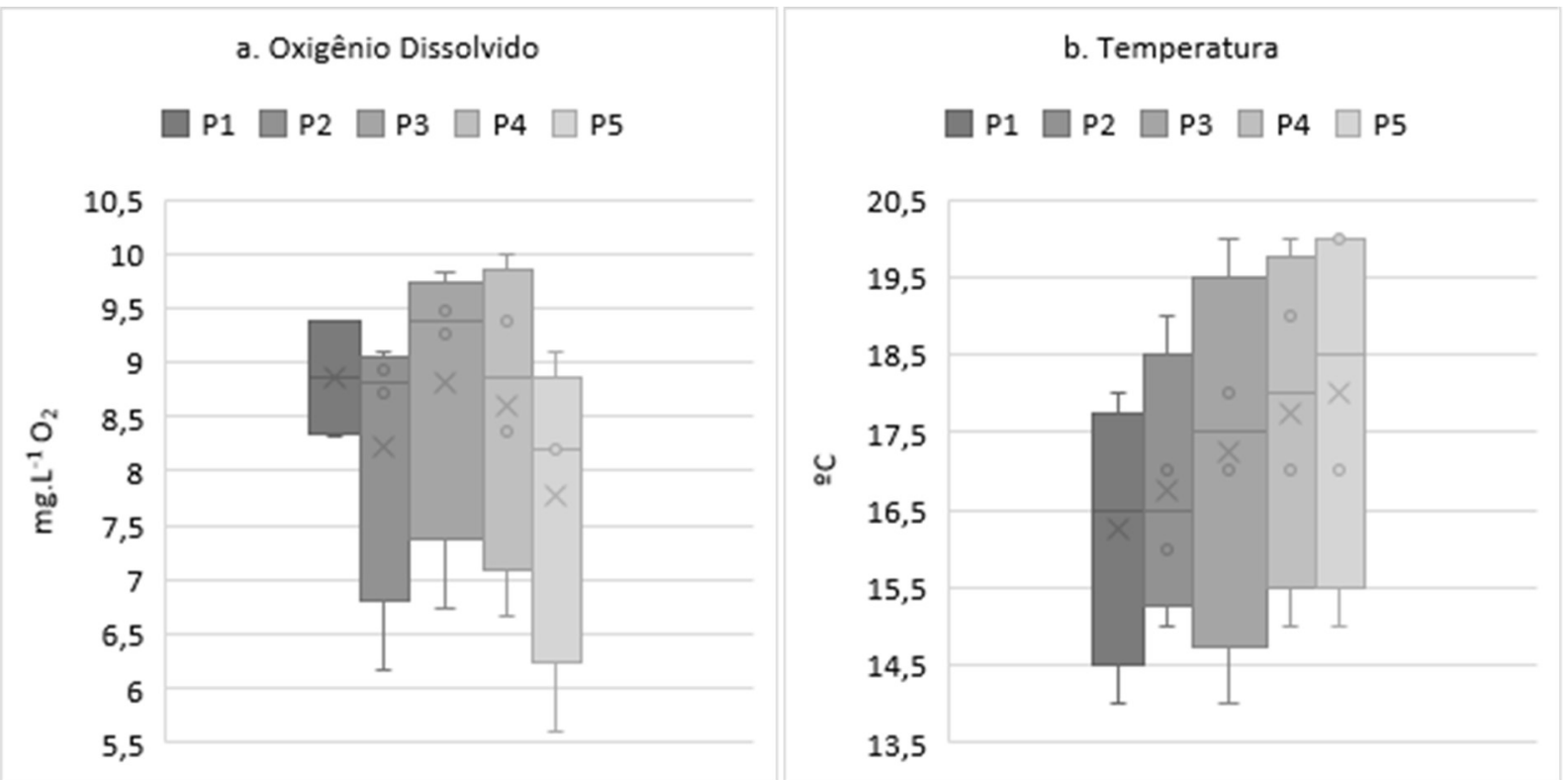

Figura 3: Box plot dos dados de oxigênio dissolvido (a) e temperatura (b) referente aos pontos de amostragem (P) da água do rio dos Índios, Presidente Getúlio (SC). Os pontos de amostragem correspondem a área rural do município (P1 e P2), limítrofe da área rural/urbana (P3) e perímetro urbano do município (P4 e P5).

A temperatura da água manteve-se em conformidade com as médias municipais de acordo com a época do ano. Contudo, é possível afirmar, a partir da análise da Figura 3b, o perímetro urbano 17.3317,33 área rural 16. Ellisson et al. (2019), afirmam que o aumento da temperatura da água do rio pode ser consequência da supressão de vegetação nativa e alteração do fluxo de água no rio (vazão), sendo que, à medida que o fluxo diminui, a temperatura aumenta. A alteração da temperatura da água pode promover grande perturbação da fauna aquática e aumento na taxa das reações químicas e biológicas resultando em mau cheiro e liberação de gases com odores desagradáveis (NIINEMETS et al., 2017).

\section{Turbidez e cor verdadeira}

A análise de dados referente aos parâmetros analíticos de turbidez e cor verdadeira não apresentaram diferenças significativas empregando o teste estatístico - teste de Tukey $(p<0,05)$ nos cinco pontos amostrais avaliados no período estudado (agosto/dezembro de 2019). Conforme estabelecido pela Resolução do CONAMA n 357/2005, o valor máximo permitido para a cor verdadeira é de $75 \mathrm{mg} \mathrm{Pt} / \mathrm{L}$ e turbidez com valor máximo de 100 UNT, para a classe 2. A Figura 4 apresenta a média dos valores da cor verdadeira e da turbidez nos pontos amostrais avaliados no curso d'água do rio dos Índios.

Os dados apontam estabilidade nos valores de turbidez (10 a 25 UNT) em todos os pontos amostrais, atendendo ao valor máximo estabelecido pela Resolução CONAMA 357/2005 (100 UNT). Contudo, do ponto de vista sanitário, o aumento da turbidez afeta esteticamente os corpos d'água e podem ser relacionados a processos erosivos devido às atividades agrícolas bem como a elevada densidade populacional em áreas urbanas que resultam no descarte de efluentes domésticos ou industriais (NASCIMENTO et al., 2019; LACH 
et al., 2020).
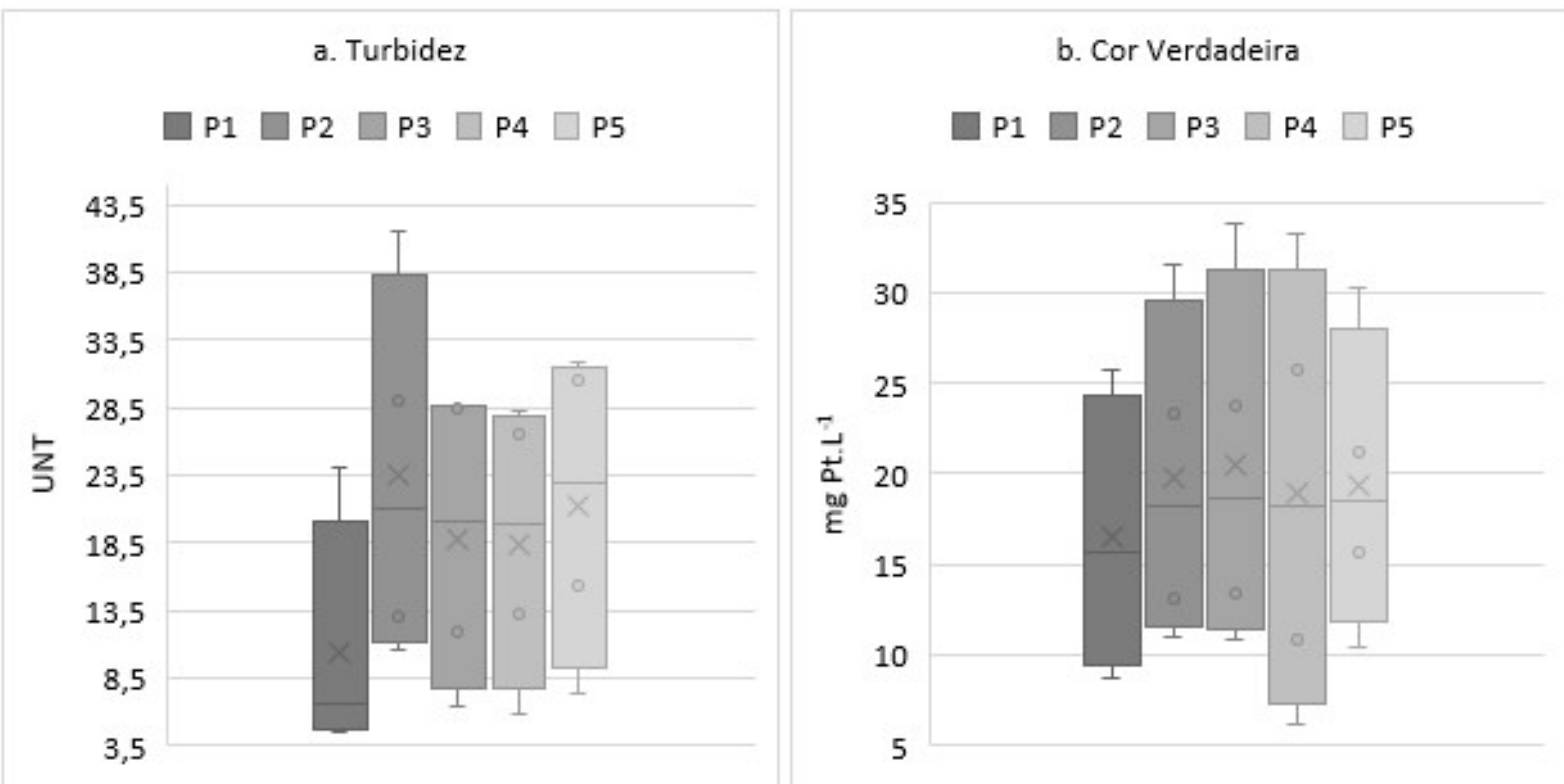

Figura 4: Box plot dos dados de turbidez (a) e cor verdadeira (b) referente aos pontos de amostragem (P) da água do rio dos Índios, Presidente Getúlio (SC). Os pontos de amostragem correspondem a área rural do município (P1 e P2), limítrofe da área rural/urbana (P3) e perímetro urbano do município (P4 e P5).

De acordo com os dados apresentados na Figura 4a, percebe-se elevação da turbidez no ponto amostral P2, localização está inserida em área de intensa produção agrícola e agropecuária. Neste sentido, um aumento na turbidez da água ocorre, principalmente, devido à presença de sólidos em suspensão e de substâncias inorgânicas que promove redução da fotossíntese de vegetação enraizada submersa e algas. Figur et al. (2017), em estudo da bacia hidrográfica do rio Abaúna em Getúlio Vargas - RS reportam uma turbidez média em torno de 14 UNT. No estudo, as autoras apontam os efeitos de ações antrópicas resultante de desmatamento e despejos de esgotos domésticos ao longo da bacia hidrográfica do rio Abaúna.

A partir da análise de dados apresentado na Figura $4 b$, foi observado um acréscimo no valor médio da cor verdadeira nos pontos de amostragem da área rural (P1: 16,33 $\mathrm{mg} \mathrm{L}^{-1} ; \mathrm{P} 2: 20 \mathrm{mg} \mathrm{L}^{-1}$ ). Desta forma, o ponto P1 apresenta melhor resultado para a cor verdadeira por estar localizado próximo à nascente do corpo hídrico e receber quantidade inferior de descarte de poluentes em relação ao ponto de amostragem P2. Marmontel et al. (2015) avaliaram a cor verdadeira da água na sub-bacia do Córrego Pimenta, com valores compreendidos na faixa de 25,80 a 93,10 mg Pt L ${ }^{-1}$, em período de estiagem, e de 19,50 a $97,90 \mathrm{mg} \mathrm{Pt} \mathrm{L} \mathrm{L}^{-1}$, em período chuvoso. Portanto, os dados corroboram com os valores obtidos para a cor verdadeira no rio dos Índios, considerando a ocorrência de baixo índice pluviométrico a época do estudo.

\section{Nitrato e nitrito}

A Figura 5 apresenta a média dos valores de nitrato e nitrito nos pontos amostrais avaliados no curso d’água do rio dos Índios.

A análise de dados referente aos parâmetros analíticos de nitrato e nitrito (Figura 5) não evidenciaram diferenças significativas empregando o teste estatístico ANOVA - teste de Tukey $(p<0,05)$ nos 
cinco pontos amostrais avaliados no período estudado (agosto/dezembro de 2019). Conforme estabelecido pela Resolução do CONAMA n 357/2005, o valor máximo permitido para o parâmetro nitrato e nitrito não deve ser superior a $10 \mathrm{mg} \mathrm{L}^{-1} \mathrm{e} 1 \mathrm{mg} \mathrm{L}^{-1}$, respectivamente para águas doces de classe 2.

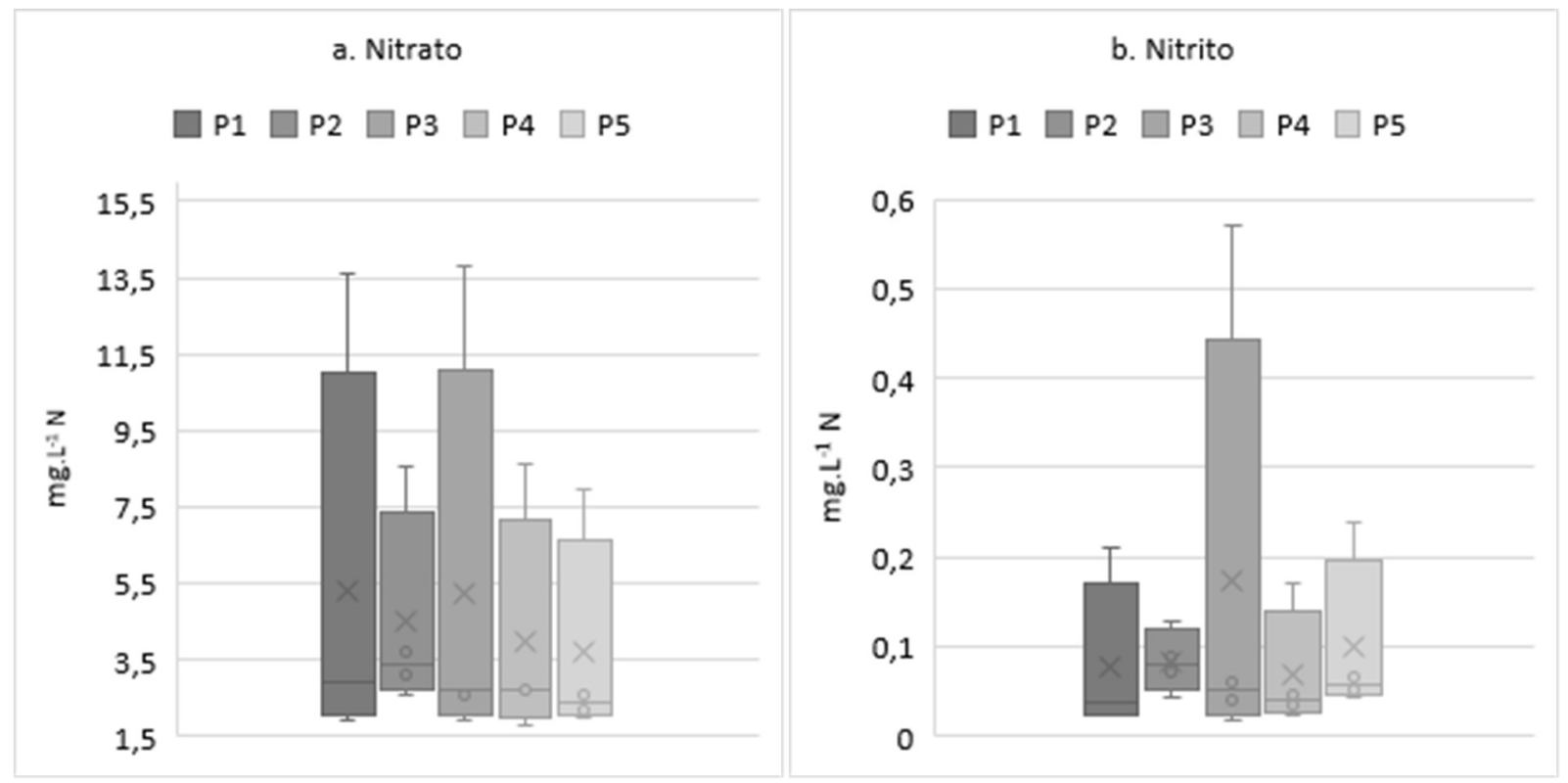

Figura 5: Box plot dos dados de nitrato (a) e nitrito (b) referente aos pontos de amostragem (P) da água do rio dos Índios, Presidente Getúlio (SC). Os pontos de amostragem correspondem à área rural do município (P1 e P2), limítrofe da área rural/urbana (P3) e perímetro urbano do município (P4 e P5).

Contudo, foi observado aumento na concentração de nitrato no mês de novembro de 2019 nos pontos amostrais P1 $\left(13,6 \mathrm{mg} \mathrm{L}^{-1}\right)$ e P3 $\left(13,6 \mathrm{mg} \mathrm{L}^{-1}\right)$, acima do limite estabelecido. Neste contexto, a poluição por nitrogênio de origem agrícola tem sido caracterizada como uma variabilidade temporal e espacial, dependendo das atividades agrícolas adotadas na região e de variáveis como clima, solo e topografia (VASCO et al., 2011). Portanto, o carreamento de partículas devido à presença de fertilizantes e insumos químicos aplicada nas culturas de fumo, milho, mandioca, feijão e arroz irrigado como sendo as mais representativas no município de Presidente Getúlio podem ser responsáveis pelo surgimento de compostos nitrogenados no período supracitado. Entretanto, todas as demais amostragens apresentaram concentrações inferiores ao estabelecido pela resolução CONAMA n 357/2005.

Analisando os resultados obtidos com referência as concentrações de nitrito na água, todos os pontos amostrais apresentaram valores inferiores ao limite máximo de $1 \mathrm{mg} \mathrm{L}^{-1}$ pela resolução CONAMA ${ }^{\circ}$ 357/2005 de nitrito para corpos d'água classe 2. Em regra, o nitrito aparece em pequenas concentrações na água, pois estes são instáveis, oxidando-se rapidamente a nitratos (CARVALHO, 2016).

\section{pH e Demanda Bioquímica de Oxigênio $\left(\mathrm{DBO}_{5}\right)$}

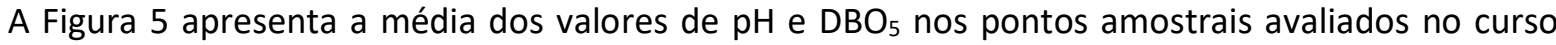
d’água do rio dos Índios. 


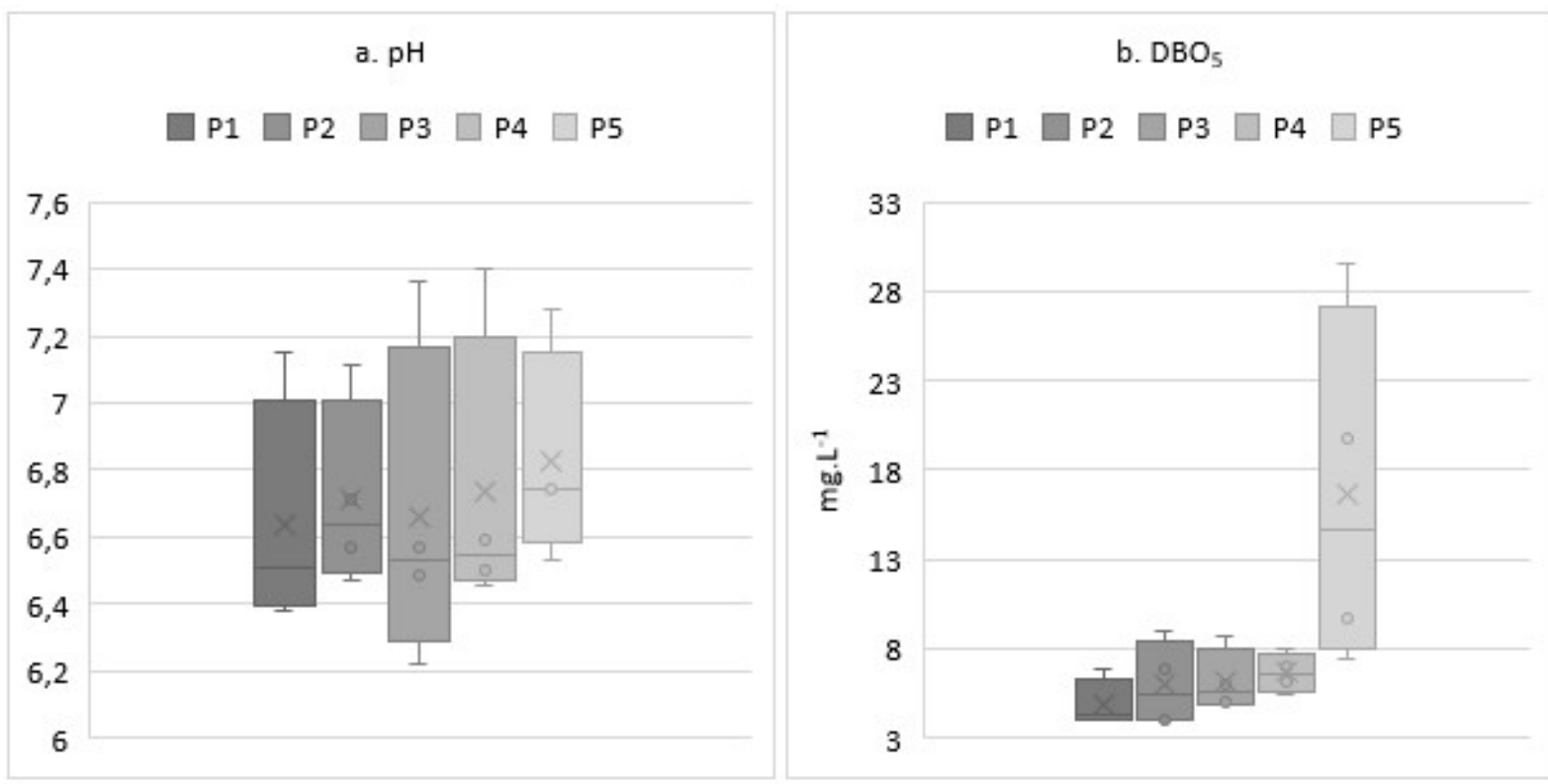

Figura 6: Box plot dos dados de $\mathrm{pH}(\mathrm{a})$ e $\mathrm{DBO}_{5}(\mathrm{~b})$ referente aos pontos de amostragem (P) da água do rio dos índios, Presidente Getúlio (SC). Os pontos de amostragem correspondem à área rural do município (P1 e P2), limítrofe da área rural/urbana (P3) e perímetro urbano do município (P4 e P5).

A análise de dados referente ao parâmetro analítico pH não evidenciou diferença significativa nos dados obtidos empregando o teste estatístico ANOVA - teste de Tukey $(p<0,05)$ nos cinco pontos amostrais avaliados no período estudado (agosto/dezembro de 2019). Conforme estabelecido pela Resolução do CONAMA n 357/2005, o valor mínimo/máximo permitido para o parâmetro pH está situada na faixa de 6,0 a 9,0 para águas doces de classe 2 .

Não obstante, a demanda bioquímica de oxigênio apresentou diferença significativa nos dados obtidos empregando o teste estatístico ANOVA - teste de Tukey $(\mathrm{p}<0,05)$. Portanto, para a $\mathrm{DBO}_{5}$ foi observado diferenças significativas em todos os pontos de amostragem exceto os localizados na área rural (P1) e (P2), ou seja, um dos possíveis fatores para a variação da $\mathrm{DBO}_{5}$ se deve a ocupação do solo da bacia hidrográfica do rio dos Índios e aos efeitos antrópicos ocasionados pelas atividades de urbanização, industrial e agroindustrial na bacia do rio dos Índios no município de Presidente Getúlio.

$\mathrm{O}$ pH é considerado um parâmetro fundamental na manutenção da fauna e flora aquática pois determina a adequação da água para diversos fins. Nesta perspectiva, conforme observado na Figura 6a, os valores de pH para o rio dos Índios apresentam tendência de oscilação levemente ácido $(6,60)$ para o levemente básico $(7,20)$, sendo que nenhum dos valores encontrados classificam-se como indicadores de risco à saúde humana. Portanto a variável pH não apresentou variação significativa de valores $\mathrm{A}$ baixa variabilidade de $\mathrm{pH}$ nas amostras é indício da reduzida atividade industrial e seus impactos sobre os pontos amostrados.

A Resolução CONAMA n 357/2005 estabelece, para corpos d'água de classe 2 , que a concentração de $\mathrm{DBO}_{5}$ não deve exceder $5 \mathrm{mg} \mathrm{L}^{-1}$, contudo, a análise de dados da Figura $6 \mathrm{~b}$ aponta valores acima do limite permitido, destacando-se o ponto amostral P5 com valor médio de $19,60 \mathrm{mg} \mathrm{L}^{-1}$. O ponto amostral P5 encontra-se localizado na área urbana do município de Presidente Getúlio/SC sendo indicativo de atividades antrópicas provenientes das atividades agrícolas, agropecuária e notadamente pelo despejo de efluentes 
domésticos. Os dados apresentados na Figura $6 \mathrm{~b}$ demonstram relativa preocupação no que tange aos valores obtidos para a $\mathrm{DBO}_{5}$ nos demais pontos amostrais conforme se segue: P1 $\left(5,19 \mathrm{mg} \mathrm{L}^{-1}\right), \mathrm{P} 2\left(6,68 \mathrm{mg} \mathrm{L}^{-1}\right), \mathrm{P} 3$ $\left(6,24 \mathrm{mg} \mathrm{L}^{-1}\right)$ e P4 $\left(6,56 \mathrm{mg} \mathrm{L}^{-1}\right)$. Neste contexto, os valores apresentados apontam a possibilidade da presença de substâncias orgânicas biodegradáveis como plantas em decomposição, materiais de origem animal, produtos químicos orgânicos, águas residuais ou urbanas e escoamento de águas pluviais.

\section{Determinação de metais}

A partir da Tabela 3, é possível verificar os valores dos pontos amostrais de todas as coletas referentes as análises da série de metais aferindo com os valores estabelecidos pela Resolução no 357/2005 do CONAMA (CONAMA, 2005).

Tabela 3: Resultados das análises de metais presentes na água e o valor de seus pontos amostrais nas quatro coletas realizadas.

\begin{tabular}{|c|c|c|c|c|c|c|c|c|c|c|c|}
\hline \multirow[t]{2}{*}{ Parâmetro } & \multicolumn{5}{|c|}{ Resultados das análises (Agosto 2019) } & \multicolumn{5}{|c|}{ Resultados das análises (Setembro 2019) } & \multirow[t]{2}{*}{ VMP(1) } \\
\hline & P1 & P2 & P3 & P4 & P5 & P1 & P2 & P3 & P4 & P5 & \\
\hline Alumínio $\left(\mathrm{mg} \mathrm{L}^{-1}\right)$ & 0,267 & 0,345 & 0,274 & 0,229 & 0,264 & 0,452 & 0,150 & 1,621 & 1,097 & 0,229 & $<0,1$ \\
\hline Manganês (mg L-1) & 0,075 & 0,104 & 0,082 & 0,027 & 0,084 & 0,086 & 0,072 & 0,079 & 0,097 & 0,083 & $<0,1$ \\
\hline $\begin{array}{l}\text { Arsênio } \\
\text { (mg L-1) }\end{array}$ & 0,023 & 0,018 & $<0,010$ & $<0,010$ & 0,020 & $<0,010$ & $<0,010$ & $<0,010$ & $<0,010$ & $<0,010$ & $<0,010$ \\
\hline Estrôncio (mg L-1) & $<0,040$ & $<0,040$ & $<0,040$ & $<0,040$ & $<0,040$ & 0,065 & 0,047 & 0,057 & 0,042 & 0,040 & $-\cdots$ \\
\hline $\begin{array}{l}\text { Ferro } \\
\left(\mathrm{mg} \mathrm{L}^{-1}\right) \\
\end{array}$ & 0,915 & 1,195 & 0,890 & 0,715 & 0,677 & 1,112 & 0,745 & 2,169 & 0,991 & 1,007 & $<0,3$ \\
\hline $\begin{array}{l}\text { Zinco } \\
\left(\mathrm{mg} \mathrm{L}^{-1}\right)\end{array}$ & 0,040 & 0,058 & 0,049 & 0,040 & 0,041 & 0,042 & 0,040 & 0,160 & 0,065 & 0,041 & $<0,18$ \\
\hline \multirow[t]{2}{*}{ Parâmetro } & \multicolumn{5}{|c|}{ Resultados das análises (Outubro 2019) } & \multicolumn{5}{|c|}{ Resultados das análises (Dezembro 2019) } & \multirow[t]{2}{*}{ VMP(1) } \\
\hline & P1 & $\mathbf{P 2}$ & P3 & P4 & P5 & P1 & $\mathbf{P 2}$ & P3 & P4 & P5 & \\
\hline Alumínio (mg L-1) & 0,270 & 0,871 & 0,723 & 0,921 & 0,628 & 0,760 & 1,369 & 1,018 & 1,001 & 0,988 & $<0,1$ \\
\hline Manganês (mg L-1) & 0,026 & 0,033 & 0,030 & 0,036 & 0,032 & 0,035 & 0,084 & 0,089 & 0,098 & 0,055 & $<0,1$ \\
\hline $\begin{array}{l}\text { Arsênio } \\
\left(\mathrm{mg} \mathrm{L}^{-1}\right)\end{array}$ & $<0,010$ & $<0,010$ & $<0,010$ & $<0,010$ & $<0,010$ & $<0,010$ & $<0,010$ & $<0,010$ & $<0,010$ & $<0,010$ & $<0,010$ \\
\hline Estrôncio (mg L-1) & $<0,040$ & $<0,040$ & $<0,040$ & $<0,040$ & $<0,040$ & $<0,040$ & $<0,040$ & $<0,040$ & $<0,040$ & $<0,040$ & $-\cdots$ \\
\hline $\begin{array}{l}\text { Ferro } \\
\left(\mathrm{mg} \mathrm{L}^{-1}\right)\end{array}$ & 1,278 & 1,942 & 1,418 & 1,592 & 1,363 & 1,264 & 2,714 & 2,014 & 3,508 & 1,650 & $<0,3$ \\
\hline $\begin{array}{l}\text { Zinco } \\
\left(\mathrm{mg} \mathrm{L}^{-1}\right)\end{array}$ & 0,041 & 0,042 & 0,042 & 0,052 & 0,043 & 0,040 & 0,042 & 0,083 & 0,050 & 0,052 & $<0,18$ \\
\hline
\end{tabular}

(1) Valor Máximo Permitido pela Resolução CONAMA n 357/2005.

Os resultados apresentados na Tabela 3 apontam para o metal alumínio valores acima do permitido pela legislação $\left(<0,1 \mathrm{mg} \mathrm{L}^{-1}\right)$ em todos os cinco pontos amostrais, sendo a menor concentração registrada no ponto próximo a nascente do Rio dos Índios (P1). Portanto, a presença de uma concentração elevada de alumínio pode ter origem da presença de indústrias do setor metal- mecânico e têxtil presente na região de estudo.

O arsênio é um metal pesado de elevada toxicidade (COSTA et al., 2018) que pode provocar diversas reações à saúde humana. As análises realizadas apontaram a presença de arsênio na primeira amostragem (agosto/2019) nos pontos P1 $\left(0,023 \mathrm{mg} \mathrm{L}^{-1}\right)$ e P2 $\left(0,018 \mathrm{mg} \mathrm{L}^{-1}\right)$ localizados na área rural do município. Deste modo, uma possível motivação pela presença deste metal na água do rio dos Índios pode ser atribuída à utilização de controle de pragas na agricultura, considerando a utilização de herbicidas aplicados em culturas de milho, uma das principais atividades econômicas do município (SANTA CATARINA, 2020). Da mesma 
forma, o ponto P5 também indicou a presença deste metal, sendo possivelmente relacionada às características do solo em torno do corpo hídrico, constituído pela associação de argissolo vermelho-amarelo e cambissolo álico (EMBRAPA, 2004), cuja configuração é representada pela presença de arsênio (SOUZA et al., 2016).

A partir da análise dos resultados da concentração de ferro na Tabela 2, observa-se em todos os pontos de amostragem valores superiores ao limite máximo definido pela legislação Conama 357/2005 para ferro total $\left(0,30 \mathrm{mg} \mathrm{L}^{-1}\right)$. A amostragem realizada no ponto $\mathrm{P} 4\left(3,508 \mathrm{mg} \mathrm{L}^{-1}\right)$ em dezembro/2019 evidenciou o maior valor encontrado no curso do rio dos Índios. Gois et al. (2016) reportaram elevada concentração de ferro em pontos de amostragem localizados em área de atividade industrial do setor metalúrgico e têxtil no curso do rio dos Índios.

Da mesma forma, as concentrações de manganês variaram na faixa de $0,026 \mathrm{mg} \mathrm{L}^{-1}$ (P1, outubro/2019) e 0,104 $\mathrm{mg} \mathrm{L}^{-1}$ (P2, setembro/2019). Sawyer et al. (2003) afirmam que o elemento manganês ocorre naturalmente em baixas concentrações nos solos, na forma de óxidos e sais pouco solúveis e, cuja solubilidade aumenta em condições redutoras, ou seja, em ambiente anaeróbico. A presença de ferro e manganês em mananciais de água superficiais ou subterrâneos destinados ao abastecimento é comum, porque esses metais estão presentes com frequência nos materiais geológicos, ocorrendo naturalmente na água pelo desgaste ou dissolução de rochas e minerais, lixiviação de solos e escoamento superficial.

O elemento estrôncio apresentou valores inferiores $\left(<0,040 \mathrm{mg} \mathrm{L}^{-1}\right)$ em todos os pontos de amostragem exceto no período de setembro/2019. Neste contexto, os valores obtidos podem ser relativos a atividades agrícolas e devida as formações geológicas constituídas for diversas rochas sedimentares com predominância de arcóseo, folhelhos, siltitos, ritmitos e arenitos ao longo do rio dos índios (BRASIL, 2014).

No presente estudo, o elemento zinco foi detectado em faixas de concentração entre de $0,040 \mathrm{mg} \mathrm{L-}$ ${ }^{1}$ e 0,164 $\mathrm{mg} \mathrm{L}^{-1}$, portanto abaixo do limite estabelecido pela Resolução Conama 357/2005 (0,18 $\left.\mathrm{mg} \mathrm{L}^{-1}\right)$. Do mesmo modo, não foi observada a presença de antimônio, cádmio, chumbo, cobalto, cromo, molibdênio, prata, tálio e vanádio em todos os ensaios realizados, sendo assim, estes não foram apresentados na tabela.

\section{CONCLUSÕES}

Estudos como esse são de extrema importância para a gestão dos recursos hídricos, sendo que, é a partir deles que os órgãos regionais conseguem avaliar a qualidade das águas de seus territórios além de determinarem quais as principais fontes de contaminação. Neste ínterim, ao analisar uma bacia hidrográfica de características rurais e urbanas ficou evidenciado que a falta de gestão dos efluentes gerados, resulta em impactos negativos a montante do ponto de lançamento, sendo isso um grave ponto a ser analisado quando levada em consideração a interferência de uma bacia hidrográfica nas demais. Para a região hidrográfica do Rio dos Índios torna-se imprescindível a otimização dos sistemas de tratamento de efluentes domésticos e industriais, uma vez que, geridos de forma inadequada ou inexistente interferem na piora da qualidade de todo o ecossistema da região. 


\section{REFERÊNCIAS}

ABNT. Associação Brasileira de Normas Técnicas. NBR 9898: preservação e técnicas de amostragem de efluentes líquidos e corpos receptores. Rio de Janeiro: ABNT, 1987.

ALVES, E. C.; SILVA, C. F.; COSSICH, E. S.; TAVARES, C. R. G.; SOUZA FILHO, E. E.; CARNIEL, A.. Avaliação da qualidade da água da bacia do Rio Pirapó - Maringá, Estado do Paraná, por meio de parâmetros físicos, químicos e microbiológicos. Acta Scientiariun Technology, v.30, n.1, p.39-48, 2008. DOI: https://doi.org/10.4025/actascitechnol.v30i1.3199

AMAVI. Associação dos Municípios do Alto Vale do Itajaí. Município de Presidente Getúlio: Representatividade por atividade econômica. Presidente Getúlio, 2018.

AMORIM, D. G.; CAVALCANTE, P. R. S.; SOARES, L. S.; AMORIM, P. E. C.. Enquadramento e avaliação do índice de qualidade da água dos igarapés Rabo de Porco e Precuá, localizados na área da Refinaria Premium I, município de Bacabeira (MA). Engenharia Sanitária e Ambiental, v.22, n.1, 2017. DOI: https://doi.org/10.1590/s1413$\underline{41522016131212}$

APHA. American Public Health Association. Standard methods for the examination of water and wastewater. 22 ed. Washington: APHA, 2012.

BARRETT, D. C.; FRAZIER, A. E.. Automated method for monitoring water quality using Landsat imagery. Water, v.8, n.6, p.1-14, 2016. DOI: https://doi.org/10.3390/w8060257

BUSTILLO-LECOMPTE, C. F.; MEHRVAR, M.. Slaughterhouse wastewater characteristics, treatment, and management in the meat processing industry: A review on trends and advances. Journal of Environmental Management, v.161, p.287-302, 2015.

BRASIL. Serviço Geológico do Brasil. Mapa geológico do estado de Santa Catarina. Porto Alegre: CPRM, 2014.

CALEGARI, R. P.; BOFFE, P.M.; PILOTO, C. A.; TESSARO, D.. Caracterização da água da microbacia do Rio Lonqueador avaliada por parâmetros físicos, químicos e microbiológicos. Revista Eletrônica em Gestão, Educação e Tecnologia Ambiental, v.19, n.2, p.1284-91, 2015. DOI: https://doi.org/10.5902/2236117016105

CARVALHO, A. P.; BALDUINO, A. R.; MACIEL, G. F.; PICANÇO, A. P.. Avaliação da poluição em rios utilizando índices de qualidade da água: um estudo de caso no ribeirão de São João em Porto Nacional/TO. Geociências, v.35, n.3, p.472484, 2016.

CREMONINI, J.; BARCAROLLI, I. F.. Análise de biomarcadores em tilápias submetidas ao efluente tratado de indústria de carne de frango. Revista Ibero-Americana de Ciências Ambientais, v.11, n.4, p.205-216, 2020. DOI: http://doi.org/10.6008/CBPC2179-6858.2020.004.0018

CONAMA. Conselho Nacional do Meio Ambiente. Resolução. 357, de 17 de março de 2005. Dispõe sobre a classificação de corpos de água e diretrizes ambientais para o seu enquadramento, bem como estabelece as condições e padrões de lançamento de efluentes e dá outras providências. CONAMA, 2005.
COSTA, A. S.; CALIMAM, T. B.; VASCONCELLOS, C. D.. Análise das alterações dos parâmetros de qualidade da água do Rio Do Carmo, afluente do rio doce, após rompimento da barragem de fundão, em Mariana-MG. Holos Environment, v.18, n.2, p.160-176, 2018. DOI:

http://doi.org/4295/holos.v18i2.12280

ISO. International Organization for Standardization. Water quality: Determination of nitrogen. Part 1: Method using oxidative digestion with peroxodisulfate. Berlin: ISSO, 1997.

EIDT, J. I.; ANDRIETTI, G.; FIGUEIREDO, L. F.; DEMARQUI, E.; ANDRADE, R. L. T.; AMARAL, A. G.; ALMEIDA, F. T.. Monitoring metals in surface water of a small watershed in amazon region. Nativa, Sinop, v.4, n.4, p.187-194, 2016. DOI: https://doi.org/10.14583/2318-7670.v04n04a01

ELLISON, J. C.; SMETHURST, P. J.; MORRISON, B. M.; KEAST, D.; ALMEIDA, A.; TAYLOR, P.; BAI, Q.; PENTON, D. J.; YU, H.. Real-time river monitoring supports community management of low-flow periods. Journal of Hydrology, v.572, p.839-850, 2019. DOI: http://dx.doi.org/10.1016/j.jhydrol.2019.03.035

EMBRAPA. Solos do Estado de Santa Catarina. Brasília: Boletim de Pesquisa e Desenvolvimento, 2004.

EPAGRI/CIRAM. Centro de Informações de Recursos Ambientais e de Hidrometeorologia de Santa Catarina. EPAGRI/CIRAM, 2020.

FATMA. Portaria estadual n.24, de 19 de setembro de 1979. Enquadra os cursos d'água do Estado de Santa Catarina. Florianópolis: DOE, 1979.

FIGUR, C.; REIS, J. T. A.. Influência do uso e cobertura da terra nos parâmetros da qualidade da água na bacia hidrográfica do rio Abaúna, em Getúlio Vargas, RS. Ciência e Natura, v.39, n.2, p.352-365, 2017.

GOIS, F. A.; SOUZA, G. A.; OLIVEIRA, M. J.; LIMA, R. S.; KOSLOWSKI, L. A. D.. Análise da qualidade da água quanto ao despejo industrial têxtil no rio dos índios. Revista Meio Ambiente e Sustentabilidade, v.9, n.5, p.15-27, 2016.

LACH, C. E.; COSTA, S. I. O.; MISTURINI, M.; UDA, Ý. F.; PAULI, C. S.; KOSLOWSKI, L. A. D.. Avaliação da qualidade da água do Rio Hercílio, município de Ibirama/SC. Revista de Ciências Ambientais, v.14, n.1, p.07-16, 2020. DOI: http://dx.doi.org/10.18316/rca.v14i1.5218

LI, H.; YE, S.; YE, J.; FAN, J.; GAO, M.; GUO, H.. Baseline survey of sediments and marine organisms in Liaohe Estuary: Heavy metals, polychlorinated biphenyls and organochlorine pesticides. Marine pollution bulletin, v.114, n.1, p.555-563, 2017.

LUNELLI, K.; NARCISO, N.B.; PAULINO, Ê. A.; KOSLOWSKI, L. A. D.. Water Quality of Rio dos Índios Under the Influence of Agro-Industrial Activities and Urbanization.Revista Virtual de Química, v.11, n.4, p.1190-1202, 2019. DOI: http://dx.doi.org/10.21577/1984-6835.20190082

MACHADO, C. S.; FREGONESI, B. M.; ALVES, R. I.; TONANI, K. 
A; SIERRA, J.; MARTINIS, B. S.; CELERE, B. S.; MARI, M.; SCHUHMACHER, M.; NADAL, M.; DOMINGO, J. L.; SEGURAMUNÕZ, S.. Health risks of environmental exposure to metals and herbicides in the Pardo River, Brazil.

Environmental Science and Pollution Research, v.24, n.25, p.20160-20172, 2017. DOI: https://doi.org/10.1007/s11356017-9461-z

MARCUZZO, F. F. N.; CARDOSO, M. R. D.; PINTO FILHO, R. F.; ROMERO, V.. Caracterização Hidromorfológica da Bacia do Vale do Itajaí. In: SIMPÓSIO BRASILEIRO DE RECURSOS HÍDRICOS, 19. Anais. Maceió, 2011.

MARMONTEL, C. V. F.; RODRIGUES, V. A.. Avaliação qualitativa de nascentes com diferentes coberturas do solo e conservação da vegetação em seu entorno no córrego Pimenta. Scientia Agraria Paranaensis, v.14, n.1, p.53-59, 2015. DOI: http://dx.doi.org/10.18188/19831471/sap.v14n1p53-59

NASCIMENTO, L. P.; REIS, D. A.; ROESER, H. M. P.; SANTIAGO, A. F.. Relationship between land use and water quality in a watershed impacted by iron ore tailings and domestic sewage. Ambiente e Agua: An Interdisciplinary Journal of Applied Science, v.14, n.5, p.1-11, 24 set. 2019. DOI: http://dx.doi.org/10.4136/ambi-agua.2383

NIINEMETS, Ü.; KAHRU, A.; MANDER, Ü.; NÕGES, P.; NÕGES, T.; TUVIKENE, A.; VASEMÄGI, A.. Interacting environmental and chemical stresses under global change in temperate aquatic ecosystems: stress responses, adaptation, and scaling. Regional Environmental Change, v.17, n.7, 20612077, 2017. DOI: https://doi.org/10.1007/s10113-017-1196$\underline{3}$

OLIVEIRA, L. F.; OLIVEIRA, B. O. S.; LIMA, L. B.. Avaliação da qualidade da água de três córregos na área urbana de Humaitá-AM. Revista de Ciências Ambientais, v.12, n.3, p.25-33, 2018. DOI:

http://dx.doi.org/10.18316/rca.v12i3.3606

OLIVEIRA, S. C.; AMARAL, R. C.; ALMEIDA, K. C. B.; PINTO, C. C.. Qualidade das águas superficiais do Médio São Francisco após a implantação dos perímetros irrigados de Gorutuba/Lagoa Grande e Jaíba. Engenharia Sanitaria e Ambiental, v.22, n.4, p.711-721, 2017. DOI: http://dx.doi.org/10.1590/s1413-41522017136784

PEREIRA, F. O. P.; SANTOS, L.; SANCHES FILHO, P. J.. Avaliação da contaminação ambiental por metais pesados, através da análise de peixes coletados da Lagoa MangueiraRS. Revista Ibero-Americana de Ciências Ambientais, v.10, n.1, p.180-194, 2019. DOI: https://doi.org/10.6008/CBPC2179-6858.2019.001.0015

PEREIRA, A. C. F.; SILVA, C. A. P.; FIGUEIREDO, F. P.; SILVEIRA, P. H. F.. Diagnóstico da qualidade ambiental de trechos do rio Pacuí, município de Coração de Jesus, norte de Minas Gerais. Revista Ibero-Americana de Ciências Ambientais, v.11, n.2, p.161-171, 2020. DOI:

https://doi.org/10.6008/CBPC2179-6858.2020.002.0018

SANTA CATARINA. Presidente Getúlio. Florianópolis, 2020.

SANTOS, L. T. S.. Caracterização de metais pesados das águas superficiais da bacia do Rio Subaé (Bahia). Geochimica Brasiliensis, v.28, n.2, p.137-148, 2015. DOI: https://doi.org/10.5327/Z0102-9800201400020003

SAWYER, C. N.; MCCARTY, P. L.; PARKIN, G. F.. Chemistry for Environmental Engineering and Science. 5.ed. Boston: McGraw-Hill, 2003.

SCOTT, A. B.; FROST, P. C.. Monitoring water quality in Toronto's urban stormwater ponds: Assessing participation rates and data quality of water sampling by citizen scientists in the FreshWater Watch. Science of the Total Environment, v.592, p.738-744, 2017. DOI:

http://doi.org/10.1016/i.scitotenv.2017.01.201

SILVA, C. V. V.; ALMEIDA, J. R.; SILVA, C. E.; CARVALHO, L. O.. Levantamento de metais pesados na água subterrânea da sub-bacia do rio Estrela, Saracuruna, Rio de Janeiro. Revista Ibero-Americana de Ciências Ambientais, v.9, n.4, p.1-11, 2018. https://doi.org/10.6008/CBPC2179$\underline{6858.2018 .004 .0001}$

SOUZA, L. C.; CAMPOS, M. L.; REICHERT, G.; MOURA, C. N.. Teores de Arsênio em solos de três regiões do estado de Santa Catarina. Ambiente \& Água: An Interdisciplinary Journal of Applied Science, Taubaté, v.11, n.1, p.135-144, 2016. DOI: http://dx.doi.org/10.4136/ambi-agua.1746

VASCO, A. N. D.; BRITTO, F. B.; PEREIRA, A. P. S.; GARCIA, C. A. B.; MÉLLO JÚNIOR, A. V.; NOGUEIRA, L. C.. Avaliação espacial e temporal da qualidade da água na sub-bacia do rio Poxim, Sergipe, Brasil. Ambiente \& Agua, Taubaté, v.6, n.1, p.118-130, 2011. DOI: http://dx.doi.org/10.4136/ambiagua.178.

VERGINE, P.; SALERNO, C.; LIBUTTI, A.; BENEDUCE, L.; GATTA, G.; BERARDI, G.; POLLICE, A.. Closing the water cycle in the agro-industrial sector by reusing treated wastewater for irrigation. Journal of Cleaner Production, v.164, p.587596, 2017. DOI:

https://doi.org/10.1016/i.jclepro.2017.06.239

YAN, Z.; YANG, H.; DONG, H.; MA, B.; SUN, H.; PAN T.; JIANG, R.; ZHOU, R., SHEN, J.; LIU, J.; LU, G.. Occurrence and ecological risk assessment of organic micropollutants in the lower reaches of the Yangtze River, China: A case study of water diversion. Environmental pollution, v.239, p.223-232, 2018. DOI: https://doi.org/10.1016/i.envpol.2018.04.023

A CBPC - Companhia Brasileira de Produção Científica (CNPJ: 11.221.422/0001-03) detém os direitos materiais desta publicação. Os direitos referem-se à publicação do trabalho em qualquer parte do mundo, incluindo os direitos às renovações, expansões e disseminações da contribuição, bem como outros direitos subsidiários. Todos os trabalhos publicados eletronicamente poderão posteriormente ser publicados em coletâneas impressas sob coordenação da Sustenere Publishing, da Companhia Brasileira de Produção Científica e seus parceiros autorizados. Os (as) autores (as) preservam os direitos autorais, mas não têm permissão para a publicação da contribuição em outro meio, impresso ou digital, em português ou em tradução. 